08

\title{
Модельные оценки квантовой емкости графеноподобных наноструктур
}

\author{
(ㄷ С.Ю. Давыдов ${ }^{1}$, А.А. Лебедев ${ }^{1}$, А.В. Зубов ${ }^{2}$, П.В. Булат ${ }^{3}$ \\ ${ }^{1}$ Физико-технический институт им. А.Ф. Иофрфе РАН, Санкт-Петербург, Россия \\ ${ }^{2}$ Университет ИТМО, Санкт-Петербург, Россия \\ ${ }^{3}$ Балтийский государственный технический университет „ВОЕНМЕХ“ им. Д.Ф. Устинова, Санкт-Петербург, Россия \\ E-mail: Sergei_Davydov@mail.ru
}

Поступило в Редакцию 21 июля 2020 г.

В окончательной редакции 7 августа 2020 г.

Принято к публикации 16 августа 2020 г.

В рамках простых моделей получены аналитические оценки квантовой емкости $C_{Q}$ для бесконечного листа, наноленты и цепочки двумерных аналогов соединений $\mathrm{A}_{N} \mathrm{~B}_{8-N}$ с гексагональной структурой (графеноподобных соединений). Продемонстрирована немонотонная зависимость $C_{Q}$ от электростатического потенциала.

Ключевые слова: бесконечный лист, наноленты, цепочка, плотность состояний.

DOI: 10.21883/PJTF.2020.23.50342.18475

Понятие квантовой емкости $C_{Q}$ впервые введено в работе [1] при исследовании двумерного электронного газа. Спустя полтора десятка лет в связи с появлением графеновой тематики возник интерес к квантовой емкости углеродных наноструктур [2-9]. Значения $C_{Q}$ были рассчитаны для однолистного $[3,5-7,9]$ и двухлистного [5,7] графенов, эпитаксиального графена на карбиде кремния [8], графеновой наноленты $[3,4,9]$ и карбина [9].

В постграфеновый период начался поиск других двумерных (2D) структур, обладающих по возможности преимуществами графена, но содержащих щель в электронном спектре. В этом плане несомненный интерес представляют 2D-аналоги бинарных полупроводников $\mathrm{A}_{N} \mathrm{~B}_{8-N}$, которые в дальнейшем будем называть графеноподобными соединениями (ГПС) [10-14]. Здесь на основе предложенных ранее простых моделей $[13,14]$ мы приведем аналитические оценки $C_{Q}$ соответственно для 2D- и одномерных (1D) наноструктур ГПС.

Определив плотность заряда $Q\left(V_{e l}\right)=e(p-n)$, наведенного внешним электростатическим потенциалом $V_{e l}$ $(e-$ величина заряда электрона, $p$ и $n-$ концентрации дырок и электронов), получим квантовую емкость $C_{Q}=\partial Q\left(V_{e l}\right) / \partial V_{e l}$. Как показано в [2], емкость $C_{Q}$ двумерной наноструктуры определяется формулой

$$
\begin{gathered}
C_{Q}=\left(e^{2} / 4 T S\right) \int_{0}^{\infty} \rho(\omega) A\left(\omega, V^{*}\right) d \omega, \\
A\left(\omega, V^{*}\right)=\operatorname{sch}^{2}\left[\left(\omega-V^{*}\right) / 2 T\right]+\operatorname{sch}^{2}\left[\left(\omega+V^{*}\right) / 2 T\right] .
\end{gathered}
$$

Здесь $\omega-$ энергетическая переменная, $V^{*}=e V_{e l}-$ сдвиг электронных состояний в электростатическом поле, $\rho(\omega)$ - плотность состояний, нормированная на один атом (здесь и далее химический потенциал совпадает с центром энергетической щели, принимаемой за начало отсчета энергии, так что $\rho(\omega)=\rho(-\omega))$, $T$ - температура в энергетических единицах, $S$ площадь, приходящаяся на один атом ГПС. Поскольку, во-первых, $\left(C_{Q}\right)_{\min } \equiv C_{Q}\left(V^{*}=0\right) \neq 0, Q\left(V^{*}=0\right)=0$ и, во-вторых, $\left(\partial C_{Q} / \partial V^{*}\right)_{V^{*}=0}=0,\left(\partial^{2} C_{Q} / \partial V^{* 2}\right)_{V^{*}=0}<0$, значение $\left(C_{Q}\right)_{\min }$ следует рассматривать как минимальную (затравочную) квантовую емкость при $V^{*} \rightarrow 0$. Если верхняя граница сплошного спектра ГПС равна $\omega_{\max }$, то при $V^{*} \gg \omega_{\max } \gg T C_{Q} \sim e^{2} / 2 T S \operatorname{ch}^{2}\left(V^{*} / 2 T\right) \rightarrow 0$, где мы учли, что для $p$-орбитали ГПС $\int_{0}^{\omega_{\max }} \rho(\omega) d \omega=1$.

Начнем с бесконечного листа ГПС, модель плотности состояний для которого в низкоэнергетическом приближении, согласно [13], имеет вид

$$
\rho_{\mathrm{GLC}}(\omega)= \begin{cases}2|\omega| / \xi^{2}, & \omega_{\max }=\sqrt{\xi^{2}+\Delta^{2}} \geq|\omega| \geq \Delta \\ 0, & |\omega|<\Delta,|\omega|>\omega_{\max }\end{cases}
$$

где $2 \Delta-$ ширина энергетической щели (запрещенной зоны), $\xi=\sqrt{2 \pi \sqrt{3} t}-$ нормировочная константа, $t-$ энергия перехода электрона между ближайшими соседями. При $\Delta=0$ выражение (4) соответствует плотности состояний графена в низкоэнергетическом приближении.

Подставляя (2) в (1), получим

$$
C_{Q}^{\mathrm{GLC}}=C I\left(x_{\max }, \delta ; v^{*}\right), \quad C=4 e^{2} T / 9 \pi a^{2} t^{2},
$$

$$
I\left(x_{\max }, \delta ; v^{*}\right)=\int_{\delta}^{x_{\max }} x A\left(x, v^{*}\right) d x
$$

где $\quad x_{\max }=\omega_{\max } / 2 T, \quad v^{*}=V^{*} / 2 T, \quad \delta=\Delta / 2 T$, $S=3 \sqrt{3} a^{2} / 4, a-$ расстояние между ближайшими 
соседями. Воспользовавшись [15], имеем

$$
\begin{aligned}
I\left(x_{\max }, \delta ; v^{*}\right)= & x_{\max }\left[\operatorname{th}\left(x_{\max }+v^{*}\right)+\operatorname{th}\left(x_{\max }-v^{*}\right)\right] \\
& -\delta\left[\operatorname{th}\left(\delta+v^{*}\right)+\operatorname{th}\left(\delta-v^{*}\right)\right] \\
& -\ln \frac{\operatorname{ch}\left(x_{\max }+v^{*}\right) \operatorname{ch}\left(x_{\max }-v^{*}\right)}{\operatorname{ch}\left(\delta+v^{*}\right) \operatorname{ch}\left(\delta-v^{*}\right)} .
\end{aligned}
$$

Из таблиц работы [13] следует, что при комнатной температуре $x_{\max } \gg 1$ и $\delta \gg 1$ для всех рассмотренных ГПС. Пусть $\left|x_{\max }-\delta\right| \gg\left|\delta-v^{*}\right| \gg 1$. Тогда

$$
I\left(x_{\max }, \delta ; v^{*}\right) \approx 4 \operatorname{ch}\left(2 v^{*}\right)\left[\delta \exp (-2 \delta)-x_{\max } \exp \left(-2 x_{\max }\right)\right]
$$

или $I\left(x_{\max }, \delta ; v^{*}\right) \sim 2 \delta \exp \left[-2\left(\delta-v^{*}\right)\right]$, так что при $\delta>v^{*}$ имеем $I\left(x_{\max }, \delta ; v^{*}\right) \ll 1$ и $\left(C_{Q}^{\mathrm{GLC}}\right)_{\min }=C \exp (-\Delta / T)$. С увеличением $v^{*}$ имеет место квадратичный рост $I\left(x_{\max }, \delta ; v^{*}\right)$, пропорциональный $I\left(x_{\max }, \delta ; v^{*}\right) \approx\left(v^{*}\right)^{2} \times$ $\times\left[\delta \exp (-2 \delta)-x_{\max } \exp \left(-2 x_{\max }\right)\right]$. Если $v^{*}>\delta$ получаем $I\left(x_{\max }, \delta ; v^{*}\right) \gg 1$, так что в области $\left|\delta-v^{*}\right| \ll 1$ с увеличением $v^{*}$ начинается линейный рост $I\left(x_{\max }, \delta ; v^{*}\right)$, пропорциональный $2\left(v^{*}-\delta\right)$. Если же $\left(v^{*}-x_{\max }\right) \gg 1$, то $I\left(x_{\max }, \delta ; v^{*}\right) \approx\left[x_{\max } \operatorname{ch}\left(2 x_{\max }\right)-\delta \operatorname{ch}(2 \delta)\right] \exp \left(-2 v^{*}\right)$ и в области $\left|v^{*}-x_{\max }\right| \ll 1$ рост $I\left(x_{\max }, \delta ; v^{*}\right)$ с увеличением $v^{*}$ сменяется экспоненциальным спадом. Таким образом, зависимость $I\left(x_{\max }, \delta ; v^{*}\right)$ от $v^{*}$ для ГПС состоит из трех характерных участков: квадратичная зависимость в области щели при $0<v^{*}<\delta$, линейная зависимость в области зоны проводимости $\left(\delta<v^{*}<x_{\max }\right)$ и экспоненциальный спад выше потолка зоны проводимости при $v^{*}>x_{\max }$. В точках $v^{*}=\delta$ и $v^{*}=x_{\max }$ происходят плавные переходы от режима к режиму. С качественной точки зрения такая же зависимость квантовой емкости от электростатического потенциала характерна и для графена [9].

Приведем значения параметров задачи для графена $(\mathrm{Gr}), \mathrm{SiC}, \mathrm{AlN}$ и $\mathrm{GaN}$ при $T=300 \mathrm{~K}$ : для $\mathrm{Gr} C=0.52 \mu \mathrm{F} / \mathrm{cm}^{2}, \quad \tau=46.0, \quad \delta=0 \quad$ [13]; для $\mathrm{SiC} C=0.80 \mu \mathrm{F} / \mathrm{cm}^{2}, \tau=29.6, \delta=29.6$ [14]; для AlN $C=0.82 \mu \mathrm{F} / \mathrm{cm}^{2}, \quad \tau=28.6, \quad \delta=48.4 \quad[16] ;$ для $\mathrm{GaN}$ $C=0.90 \mu \mathrm{F} / \mathrm{cm}^{2}, \tau=26.3, \delta=38.7$ [16].

Рассмотрим вырезанную из ГПС наноленту с зигзагообразными краями (ZNR), зонная структура которой достаточно подробно обсуждается в $[14,16]$. Учитывая только низкоэнергетические „Плоские“ зоны, определяющие ширину запрещенной зоны $E_{g}=2 t$, плотности состояний зоны проводимости и валентной зоны можно соответственно аппроксимировать выражениями $\rho_{\mathrm{ZNR}}(\omega) \sim \delta(\omega-t)$ и $\rho_{\mathrm{ZNR}}(\omega) \sim \delta(\omega+t)$, где $\delta(\ldots)-$ функция Дирака, начало отсчета энергии помещено в центр щели. Тогда из (1) получаем

$$
\begin{gathered}
C_{Q}^{\mathrm{ZNR}} \sim C^{\prime} \bar{A}, \quad C^{\prime}=e^{2} / 3 \sqrt{3} T a^{2}, \\
\bar{A}=\operatorname{sch}^{2}\left[\left(t-V^{*}\right) / 2 T\right]+\operatorname{sch}^{2}\left[\left(t+V^{*}\right) / 2 T\right] .
\end{gathered}
$$

Переходя к введенным выше безразмерным единицам, при $\left(\tau-v^{*}\right) \gg 1$ получаем $\bar{A} \approx 8 \operatorname{ch}\left(2 v^{*}\right) \exp (-2 \tau)$, откуда следует, что $\bar{A} \propto\left(v^{*}\right)^{2}$ при $v^{*} \ll 1$. Если $\left(v^{*}-\tau\right) \gg 1$, то $\bar{A} \approx 8 \operatorname{ch}(2 \tau) \exp \left(-2 v^{*}\right)$. Кроссовер имеет место при $v^{*} \sim \tau$. Отметим, что для ZNR множитель $C^{\prime} \propto T^{-1}$, тогда как для листа ГПС множитель $C \propto T$ (см. (3))

Рассмотрим теперь двухатомную цепочку, спектр состояний которой имеет вид $E_{ \pm}(k)= \pm \sqrt{\Delta^{2}+t^{2} \Phi}$, где $\Phi=4 \cos ^{2}(k a), k-$ волновой вектор для движения электрона вдоль цепочки, за нуль энергии принят центр щели. Функция Грина элементарной ячейки двухатомной цепочки равна

$$
C_{\text {chain }}(\omega, k)=\frac{2(\omega-\Delta)}{\left(\omega-E_{+}(k)+i 0^{+}\right)\left(\omega-E_{-}(k)+i 0^{+}\right)} .
$$

В низкоэнергетическом приближении получаем $E(k) \approx \pm \Delta\left(1+2 t^{2} a^{2} q^{2} / \Delta^{2}\right)$, где $q=(\pi / a)-k$ и $q a \ll 1$. Тогда плотность состояний для элементарной ячейки можно представить в виде

$$
\rho_{\text {chain }}(\omega)=\frac{2}{\pi^{2} \varepsilon \Delta}\left(\frac{1}{2} \ln \left|\frac{\varepsilon+y_{c}}{\varepsilon-y_{c}}\right|+\operatorname{arctg} \frac{y_{c}}{\varepsilon}\right),
$$

где $\varepsilon=\sqrt{\Delta(|\omega|-\Delta) / 2 t^{2}}, y_{c}=a q_{c}, q_{c}-$ волновой вектор обрезания. Поскольку выражение в скобках сравнительно слабо зависит от параметра $\varepsilon$, можно приближенно аппроксимировать плотность состояний как $\rho_{\text {chain }}(\omega) \approx B / \varepsilon \Delta$, где $B=(\Delta / t) \sqrt{\Delta / 2 W}-$ нормировочный множитель. Тогда можем записать окончательно

$$
\begin{gathered}
\rho_{\text {chain }}(\omega) \approx \begin{cases}\frac{1}{\sqrt{W(|\omega|-\Delta)}}, & \Delta \leq|\omega| \leq W, \\
0, & |\omega|<\Delta,\end{cases} \\
C_{Q}^{\text {chain }}=\left(e^{2} / 8 T a\right) J\left(w, \delta ; v^{*}\right), \\
J\left(w, \delta ; v^{*}\right)=\int_{\delta}^{w+\delta} \rho_{\text {chain }}(x) A\left(x, v^{*}\right) d x,
\end{gathered}
$$

где $w=W / 2 T$ и площадь $S$ заменена постоянной цепочки 2a. При $\left|\delta-v^{*}\right| \gg 1$ положим $J\left(v^{*}\right)=16 \operatorname{ch}\left(2 v^{*}\right) \exp (-2 \delta)$, так что

$$
C_{Q}^{\text {chain }} \sim\left(4 e^{2} / T a\right) \exp \left(-\left|\Delta-V^{*}\right| / T\right) .
$$

Вновь при $V^{*} \sim \Delta$ имеет место кроссовер. При $V^{*} / 2 T \ll 1$ имеем

$$
C_{Q}^{\text {chain }} \sim\left(4 e^{2} / T a\right)\left(1+V^{*} / T\right) \exp (-\Delta / T) .
$$

Отметим следующее обстоятельство. Поскольку ГПС можно отнести к полупроводниковым (или диэлектрическим) соединениям, можно было бы сопоставить полученные результаты с результатами работ по емкостным характеристикам квазидвумерных объектов - тонких полупроводниковых пленок (ТПП) (см. обзор [17], а также работы $[18,19]$ и ссылки, приведенные в них). 
Однако сходство ГПС и ТПП в значительной степени иллюзорно. Во-первых, в ТПП вследствие конечной толщины пленки и пространственного квантования вдоль оси $z$, перпендикулярной плоскости пленки, возникает ряд $z$-подзон, тогда как ГПС является монослойной структурой и содержит лишь одну $z$-зону. Во-вторых, в ТПП электронный спектр имеет параболический или кейновский (см. [19]) характер, в то время как у ГПС спектр линеен.

Итак, в настоящей работе впервые, насколько известно авторам, сделаны оценки квантовой емкости для ГПС. Для проверки приемлемости использованных в работе моделей необходимы данные экспериментов по зависимости квантовой емкости ГПС от $V_{e l}$ и $T$. Определенную ясность могли бы внести и расчеты из первых принципов.

\section{Финансирование работы}

П.В. Булат и А.А. Лебедев благодарны за финансовую поддержку Министерству науки и высшего образования РФ в ходе реализации проекта „Фундаментальные основы механики, систем контроля и управления беспилотных авиационных систем с формообразующими конструкциями, глубоко интегрированными с силовыми установками, и уникальными свойствами, не применяемыми сегодня в пилотируемой авиации“ (№ FEFM-2020-0001).

\section{Конфликт интересов}

Авторы заявляют, что у них нет конфликта интересов.

\section{Список литературы}

[1] Luryi S. // Appl. Phys. Lett. 1988. V. 96. N 6. P. 501-503.

[2] John D.L., Castro L.C., Pulfrey D.L. // J. Appl. Phys. 2004. V. 96. N 9. P. 5180-5184.

[3] Fang T., Konar A., Xing H., Jena D. // Appl. Phys. Lett. 2007. V. 91. N 9. P. 092109.

[4] Guo J., Yoon Y., Ouyang Y. // Nano Lett. 2007. V. 7. N 7. P. $1935-1940$.

[5] Kliros G.S. // Romanian J. Inform. Sci. Technol. 2010. V. 13. N 3. P. $332-341$.

[6] Cheremisin M.V. // Physica E. 2015. V. 69. N 1. P. 153-158.

[7] Alisultanov Z.Z., Reis M.S. // EPL. 2016. V. 113. N 2. P. 28004.

[8] Trabelsi A.B.G., Kusmartsev F.V., Forrester D.M., Kusmartseva O.E., Gaifullin M.B., Cropper P., Oueslati M. // J. Mater. Chem. C. 2016. V. 4. N 24. P. 5829-5838.

[9] Давыдов С.Ю., Лебедев А.А., Булат П.В., Зубов А.В. // Письма в ЖТФ. 2020. Т. 46. В. 15. С. 7-9.

[10] Tong C.-J., Zhang H., Zhang Y.-N., Liu H., Liu L.-M. // J. Mater. Chem. A. 2014. V. 2. N 42. P. 17971-17978.

[11] Singh A.K., Zhuang H.L., Hennig R.G. // Phys. Rev. B. 2014. V. 89. N 24. P. 245431.

[12] Kecik D., Onen A., Konuk M., Gürbüz E., Ersan F., Cahangirov S., Aktürk E., Durgun E., Ciraci S. // Appl. Phys. Rev. 2018. V. 5. N 1. P. 011105.
[13] Давыдов С.Ю. // ФТТ. 2016. Т. 58. В. 4. С. 779-790.

[14] Давыдов С.Ю. // ФТП. 2020. Т. 54. В. 5. С. 446-451.

[15] Градштейн И.С., Рыжик И.М. Таблицы интегралов, сумм, рядов и произведений. М.: Наука, 1971. 1108 с.

[16] Давыдов С.Ю. // ФТТ. 2020. Т. 62. В. 6. С. 955-959.

[17] Ando T., Fowler A.B., Stern F. // Rev. Mod. Phys. 1982. V. 54. N 2. P. 438-672.

[18] Цуриков Д.Е., Ябясов А.М. // ФТП. 2013. Т. 47. В. 9. C. $1169-1174$.

[19] Дубицкий И.С., Ябясов А.М. // ФТП. 2014. Т. 48. В. 3. C. $327-333$. 